\title{
REACTION DEPENDENCE OF NUCLEAR DECAY LINEWIDTHS
}

\author{
D. OVERWAY and J. JÄNECKE ${ }^{\dagger}$ \\ Department of Physics, University of Michigan, Ann Arbor, Michigan 48109, USA \\ F. D. BECCHETTI \\ Department of Physics, University of Michigan, Ann Arbor, Michigan 48109, USA \\ and \\ Hahn-Meitner Institute ${ }^{\dagger+}$, Berlin 39, W. Germuny \\ C. E. THORN \\ Department of Physics, Brookhaven National Laboratory ${ }^{\ddagger}$, Upton, LI, NY, 11973, USA \\ and \\ G. KEKELIS \\ Department of Physics and Astronomy, Williams College, Williamstown, Mass. 01267, USA
}

Received 30 October 1980

Abstract: Various light- and heavy-ion reactions, $20<E<100 \mathrm{MeV}$, have been used to study the reaction dependence of $\alpha$-decay widths for ${ }^{8} \mathrm{Be}^{*}\left(2^{+}, 2.9 \mathrm{MeV}\right)$ and ${ }^{16} \mathrm{O}^{*}\left(1^{-}, 9.6 \mathrm{MeV}\right)$. Although slight differences $(<20 \%)$ are found for the observed line shapes $(\Gamma)$, the resonance widths inferred $\left(\Gamma_{\mathrm{R}}\right)$ are self-consistent and indicate little if any reaction dependence $(<10 \%)$. Near a decay threshold one may expect $\Gamma<\Gamma_{\mathrm{R}}$ by $20 \%$ or morc, however, and thus carc must be taken in comparing decay widths inferred from nuclear reactions with those from scattering resonances. Reduced formal $\alpha$-decay widths of $\gamma_{\lambda}^{2}=680 \pm 100 \mathrm{keV}(s=4.8 \mathrm{fm})$ and $\gamma_{\lambda}^{2}=350 \pm 50 \mathrm{keV}(s=5.4$ fm), corresponding to $\theta_{\lambda}^{2}=0.50$ and $\theta_{\lambda}^{2}=0.49$ are deduced for ${ }^{8} \mathrm{Be}^{*}\left(2^{+} .2 .9 \mathrm{MeV}\right)$ and ${ }^{16} \mathrm{O}^{*}\left(1^{-}, 9.6\right.$ $\mathrm{MeV})$ using the nuclear-reaction $\Gamma_{\mathrm{R}}$ values and a particular set of $\alpha$-nucleus potentials.

NUCLEAR REACTIONS ${ }^{11} \mathrm{~B}(\mathrm{p}, \alpha),{ }^{9} \mathrm{Be}(\mathrm{p}, \mathrm{d}),{ }^{12} \mathrm{C}\left(\mathrm{d},{ }^{6} \mathrm{Li}\right), E=33 \mathrm{MeV} ;{ }^{9} \mathrm{Be}(\mathrm{d}, \mathrm{t})$, ${ }^{19} \mathrm{~F}(\mathrm{p}, \alpha), E=26 \mathrm{MeV} ;{ }^{12} \mathrm{C}\left({ }^{12} \mathrm{C},{ }^{16} \mathrm{O}\right), E=80 \mathrm{MeV} ;{ }^{12} \mathrm{C}\left({ }^{6} \mathrm{Li}, \alpha\right), E=42,90 \mathrm{MeV}$; ${ }^{12} \mathrm{C}\left({ }^{7} \mathrm{Li}, \mathrm{t}\right), E=34 \mathrm{MeV} ;{ }^{12} \mathrm{C}\left({ }^{10} \mathrm{~B},{ }^{6} \mathrm{Li}\right), E=73 \mathrm{MeV}$; measured $\sigma\left(E_{\mathrm{a}}\right), \sigma\left(E_{\mathrm{d}}\right), \sigma\left(E_{\mathrm{t}}\right)$, $\sigma\left(E\left({ }^{6} \mathrm{Li}\right), \quad \sigma\left(E\left({ }^{16} \mathrm{O}\right) .{ }^{8} \mathrm{Be},{ }^{16} \mathrm{O}\right.\right.$ resonances deduced $\Gamma, \alpha$-reduced widths.

$\dagger$ Supported in part by the National Science Founciation. (Grant Phy 78-07754).

${ }^{\dagger+}$ Supported by the German Federal Republic Science Foundation.

* Supported by the US Department of Energy. 


\section{Introduction}

Nuclcar-decay widths, $\Gamma$, deduced from the observed line shapes in various nuclear reactions often differ with each other and those inferred from resonant scattering and other experiments. The presence of a third particle in the vicinity of a nucleus can introduce interactions which, in principle, can alter the intrinsic decay of nuclear states, particularly if the particle's transit time is comparable to the decay lifetime. The presence of the third particle may increase the apparent lifetime, $t$, of the nuclear state formed beyond that of the isolated intrinsic state, $t_{0}$, with e.g. $t \approx t_{0}+t_{\mathrm{r}}$ where $t_{\mathrm{r}}$ is the reaction time. Such phenomena have been observed in atomic ${ }^{1,2}$ ) and elementary particle physics ${ }^{3}$ ).
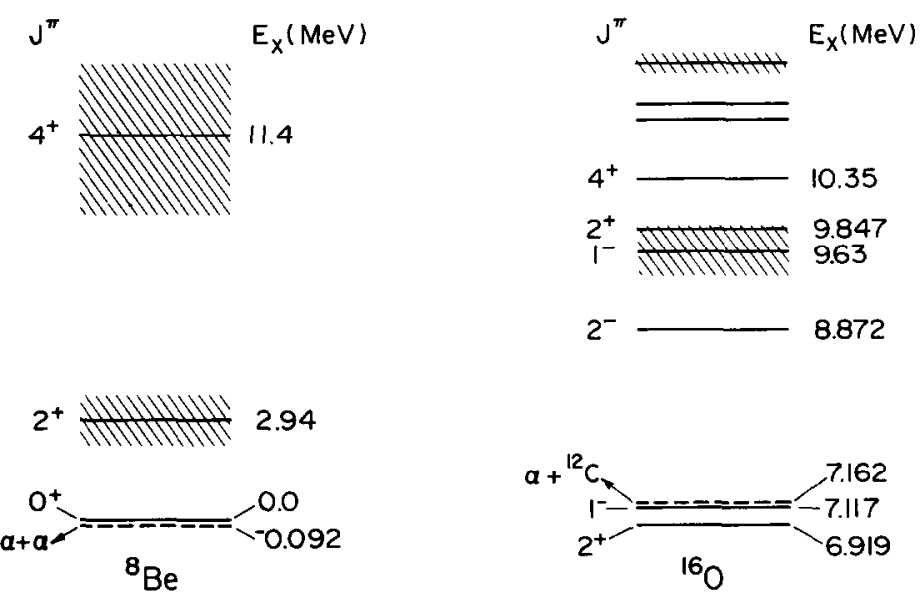

Fig. 1. The spin, parity $\left(J^{n}\right)$, and exitation energy $\left(E_{\mathrm{x}}\right)$ of levels in ${ }^{8} \mathrm{Be}$ and ${ }^{16} \mathrm{O}$ near their $\alpha$-decay thresholds ${ }^{6}$ ).

The nuclei ${ }^{8} \mathrm{Be}$ and ${ }^{16} \mathrm{O}$ both exhibit levels (fig. 1) which are above $\alpha$-decay thresholds yet below other particle decay modes. The levels at $E_{\mathrm{x}} \approx 2.9$ and 9.6 $\mathrm{MeV}$, respectively, have widths $(\Gamma \approx 1 \mathrm{MeV})$ corresponding to decay lifetimes comparable to the ion transit time in a typical nuclear reaction $\left(10^{-22} \mathrm{~s}\right)$. These nuclei are therefore well suited for study of reaction-time or other related effects.

Berkowitz $\left.{ }^{4}\right)$ in a survey of nuclear decay widths measured for ${ }^{8} \mathrm{Be}^{*}\left(J^{\pi}=2^{+}\right.$, $E_{\mathrm{x}}=2.9 \mathrm{MeV}$ ) reported an apparent correlation between $\Gamma$ and the relative velocity, $v_{\text {rel }}$, of the ejectile, in qualitative agreement with the expected behavior: $t-t_{0} \propto$ $\Gamma^{-1}-\Gamma_{0}^{-1} \propto t_{\mathrm{r}} \propto v_{\mathrm{rel}}^{-1}$. This early analysis was later contradicted by measurements and analysis of Nüsslin et al. ${ }^{5}$ ) who found little, if any, correlation between $\Gamma$ and $v_{\text {rel}}$, at least for levels in ${ }^{8} \mathrm{Be}$. Both of these analyses utilized primarily light-ion data obtained at low bombarding energies $(E<20 \mathrm{MeV})$. More recent experiments, again with light ions, have yielded values for $\Gamma\left({ }^{8} \mathrm{Be}^{*}, 2.9 \mathrm{MeV}\right)$ from $0.9 \mathrm{MeV}$ to $1.8 \mathrm{MeV}$ [ref. $\left.\left.{ }^{6}\right)\right]$. (Unless otherwise noted all $\Gamma$-values are in the c.m.s.)

Additional interest in this problem stems from studies of $\alpha$-transfer reactions on 
${ }^{12} \mathrm{C}$ forming levels in ${ }^{16} \mathrm{O}\left(\mathrm{J}^{\pi}=1^{-}, E_{\mathrm{x}}=7.1\right.$ and $\left.9.6 \mathrm{MeV}\right)$ which are relevant to stellar helium burning. The apparent decay width $\Gamma\left(1^{-}, 9.6 \mathrm{MeV}\right)$ observed [refs. $\left.{ }^{7.8}\right)$ ] with ${ }^{12} \mathrm{C}\left({ }^{6} \mathrm{Li}, \mathrm{d}\right)$ and ${ }^{12} \mathrm{C}\left({ }^{7} \mathrm{Li}, \mathrm{t}\right)$ was found to be less $(\Gamma \approx 400 \mathrm{keV})$ than the value based on $\left({ }^{3} \mathrm{He}, \mathrm{d}\right)$ and $(\alpha, \alpha)$ measurements $\left.{ }^{6}\right)(\Gamma \approx 510 \mathrm{keV})$. Subsequent measurements $\left.{ }^{9}\right)$ employing the heavy-ion reaction $\left({ }^{10} \mathrm{~B},{ }^{6} \mathrm{Li}\right)$ yielded $\Gamma\left(1^{-}, 9.6 \mathrm{MeV}\right)$ $\approx 320 \mathrm{keV}$, and suggest a possible reaction dependence of the observed $\Gamma$.

Heavy ions are now extensively employed in the formation and study of nuclear levels, particularly those in the continuum such as giant resonances. Should extraction of properties such as intrinsic level widths depend strongly on the reaction time (or e.g. the mass transfer) much of the data obtained with heavy ions could be affected since $v_{\text {rel }}$ (and the mass transfer) can vary substantially among heavyion reactions.

In addition to possible reaction-time dependence, one has other complications that can affect observed line widths. These include penetrability and kinematic effects, quantum interference between continuum or bound levels of the same $J^{\pi}$, interference with projectile break-up, etc. Destructive quantum interference has been observed recently for neutron stripping to unbound levels ${ }^{10.11}$ ). The effect is often to narrow the apparent line width of the level and is similar to the phenomena discussed by Fano $^{2}$ ) for atomic collisions.

Barker ${ }^{12}$ ) has suggested that bound states or levels near a decay threshold can produce features in the continuum which resemble broad levels as is observed for the deuteron ${ }^{13}$ ). A similar feature may exist just above the ${ }^{8} \mathrm{Be}$ g.s. and has been studied extensively by Barker and his co-workers ${ }^{12,14}$ ). Berkowitz et al. ${ }^{15}$ ) and Loren $\mathrm{z}^{16}$ ) propose that the phenomenon observed in ${ }^{8} \mathrm{Be}$ can be attributed to three-body phase-space thresholds. The latter is common in elementary particle physics ${ }^{3}$ ). The anomaly in ${ }^{8} \mathrm{Be}$ described by Barker should exhibit characteristics that become pronounced at high bombarding energies $(E>20 \mathrm{MeV})$.

\section{Experiments}

The reactions chosen for study are listed in table 1 . The majority of the measurements utilized the BNL tandem Van de Graaff accelerator and QDDD magnetic spectrometer. Reaction products were identified with resistive-wire proportional counters, 60 and $90 \mathrm{~cm}$ in length, which employed charge division to yield ion position. The $B \rho$ versus position calibration of the spectrometer-detector system was determined with elastically scattered ions or reaction products from transitions to bound levels.

Several of the previous ${ }^{12} \mathrm{C}\left({ }^{6} \mathrm{Li}, \mathrm{d}\right){ }^{16} \mathrm{O}$ measurements $\left.{ }^{7}\right)$ at $E\left({ }^{6} \mathrm{Li}\right)=42 \mathrm{MeV}$ were repeated with larger detectors to facilitate multi-peak fitting and background determination. Data for $\left.{ }^{12} \mathrm{C}^{7} \mathrm{Li}, \mathrm{t}\right)^{16} \mathrm{O}$ are taken from ref. $\left.{ }^{8}\right)$ and were not rcmeasured. Additional ${ }^{12} \mathrm{C}\left({ }^{6} \mathrm{Li}, \mathrm{d}\right){ }^{16} \mathrm{O}$ data were obtained for $E\left({ }^{6} \mathrm{Li}\right)=90 \mathrm{MeV}$ at the Indiana University Cyclotron Facility with a magnetic spectrometer and helical-wire counter. This experiment has been reported in detail elsewhere ${ }^{17}$ ). 


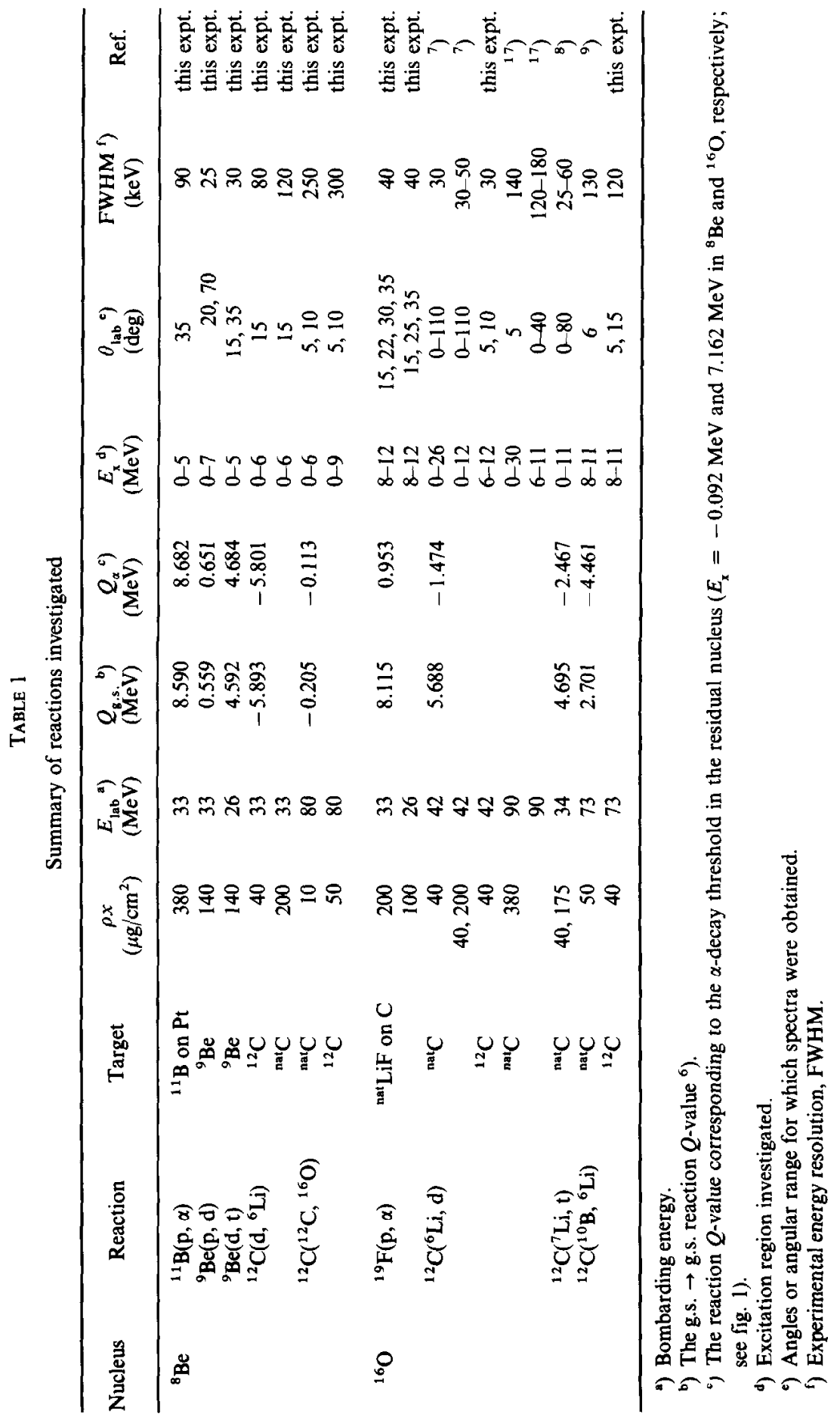




\section{Spectra}

The original spectra, which were obtained as a function of magnetic rigidity, have been transformed to spectra linear in the c.m. excitation of the residual system using the aforementioned calibrations. The results are displayed in figs. 2 to 11 for ${ }^{8} \mathrm{Be}^{*}$ and ${ }^{16} \mathrm{O}^{*}$.

\subsection{ANALYSIS WITH FIXED WIDTHS}

The data were subsequently analysed with a multi-peak fitting computer program [ref. $\left.\left.{ }^{18}\right)\right]$. Initial fits were performed with simple lorentzian and gaussian shapes with energy-independent fixed line widths together with appropriate exponentialtype background terms.

The lorentzian peak shape, $N_{\mathbf{L}}(E)$, is given by

$$
N_{\mathrm{L}}(E) \propto \frac{\Gamma}{\left(E-E_{\mathrm{c}}\right)^{2}+\left(\frac{1}{2} \Gamma\right)^{2}},
$$

where $\Gamma$ is the FWHM, $E_{\mathrm{c}}$ is the centroid energy and $E$ is the excitation energy in the c.m.s. In principle one must convolute $N_{\mathrm{L}}(E)$ with the experimental resolution before extracting $\Gamma$ and $E_{\mathrm{c}}$. Since the decay line widths were usually much greater than the experimental line widths (table 1) exact numerical convolution was not performed. Rather, the experimental line widths were subsequently extracted

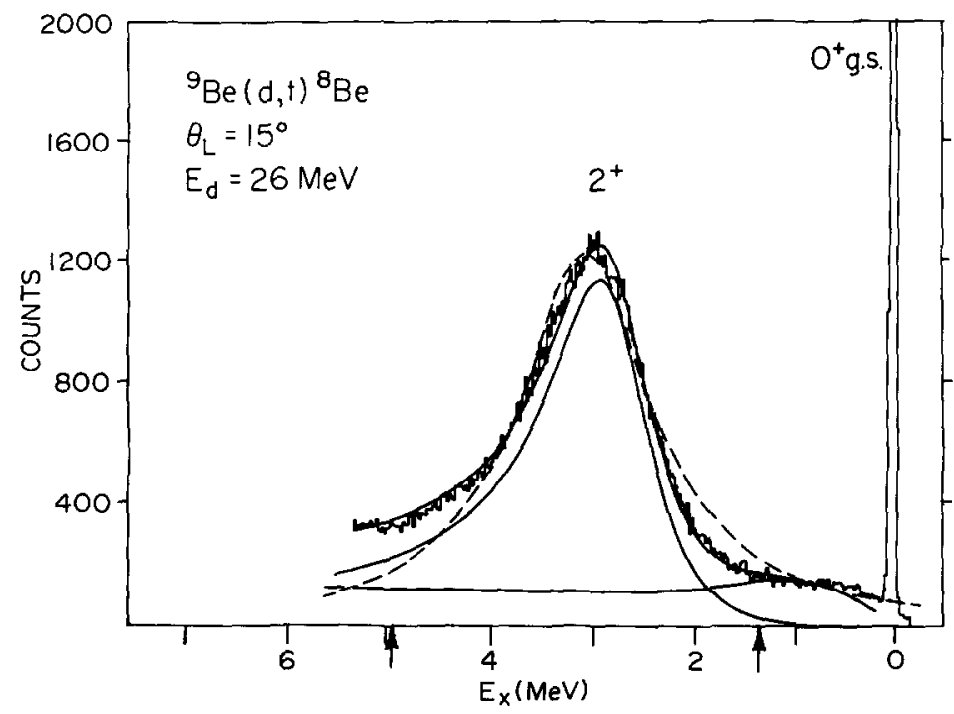

Fig. 2. Data and line-shape fits for ${ }^{9} \mathrm{Be}(\mathrm{d}, \mathrm{t})^{8} \mathrm{Be}$. The solid curve represents a fit utilizing an energydependent width with $\Delta(E)=0$ [eqs. (6) to (8)]. The broken curve represents a fit with an energy-independent width $[\Gamma$, eq. (I)]. The region included in the fit is indicated by vertical markers. 


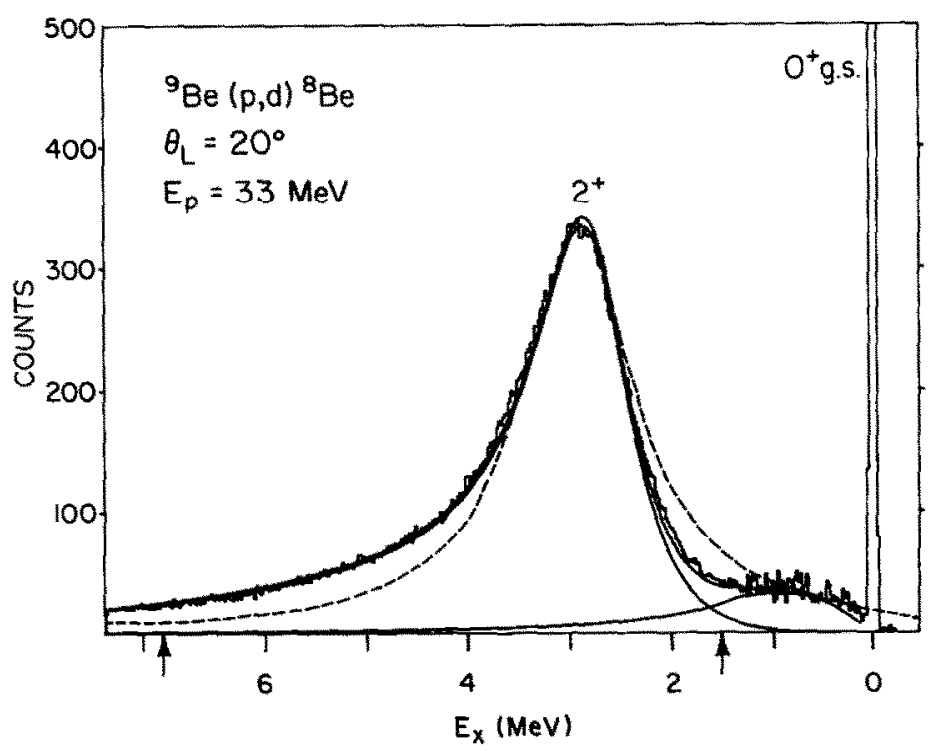

Fig. 3a. Same as fig. 2, for ${ }^{9} B e(p, d)$ at $\theta=20^{\circ}$.

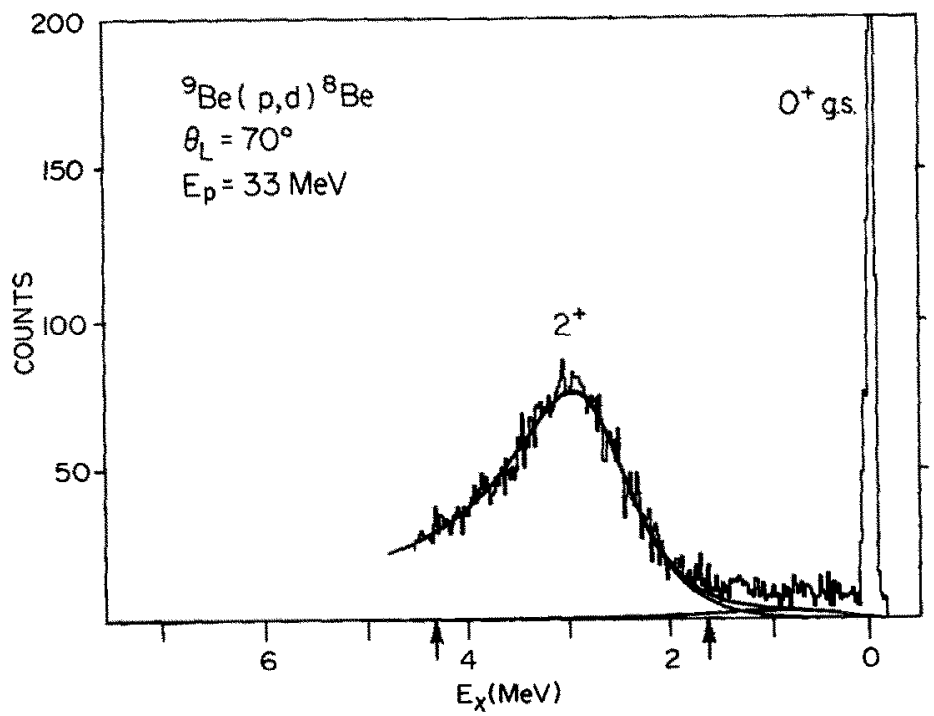

Fig. 3b. Same as fig. 2, for ${ }^{9} \mathrm{Be}\left(\mathrm{p}\right.$, d) at $\theta=70^{\circ}$. 


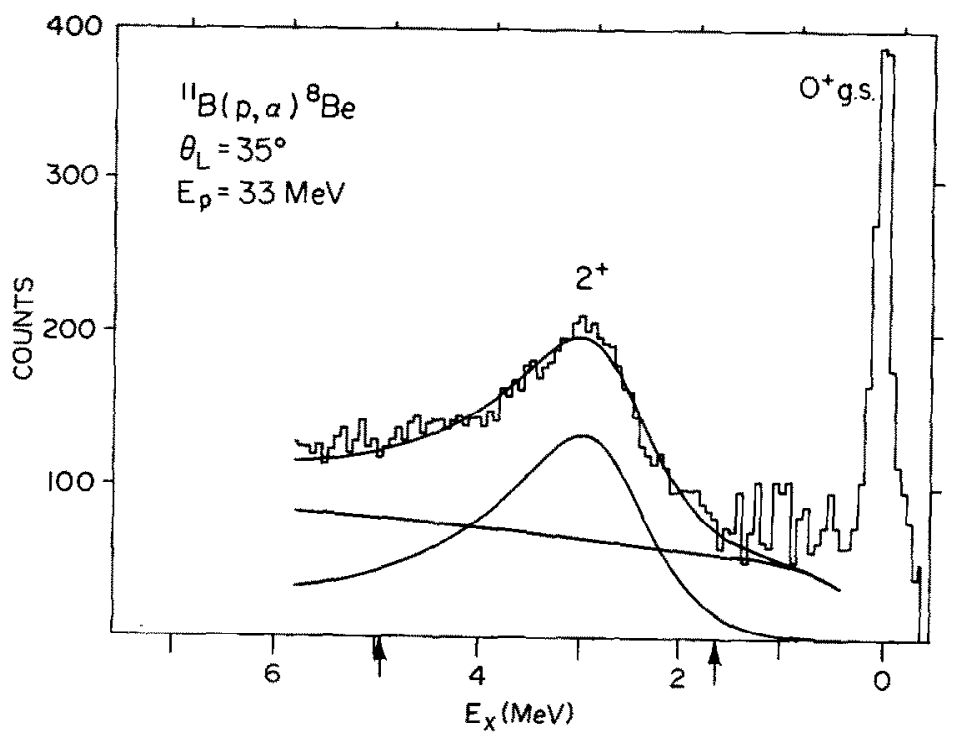

Fig. 4. Same as fig. 2, for ${ }^{11} B(p, \alpha)$.

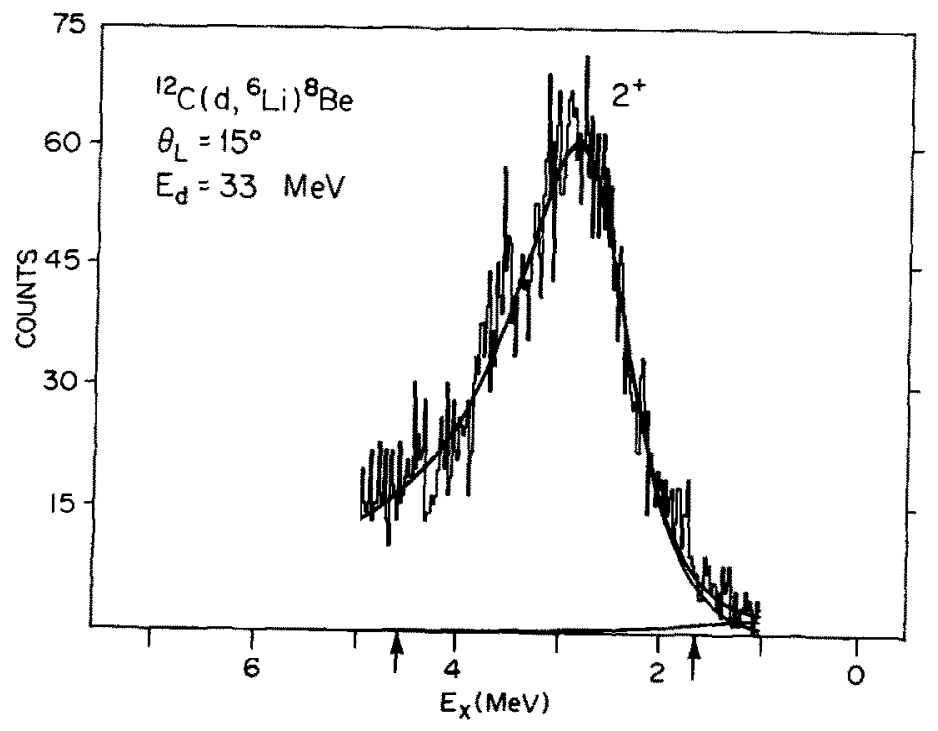

Fig. 5. Same as fig. 2 , for ${ }^{12} \mathrm{C}\left(\mathrm{d},{ }^{6} \mathrm{Li}\right)$. 


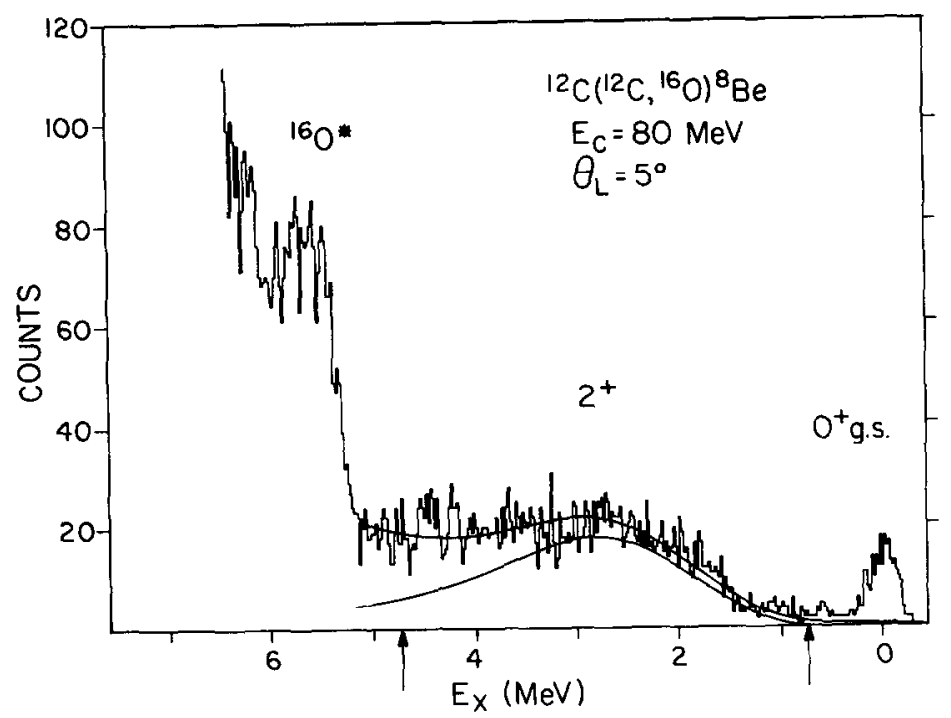

Fig. 6. Same as fig. 2 , for ${ }^{12} \mathrm{C}\left({ }^{12} \mathrm{C},{ }^{16} \mathrm{O}\right)$.

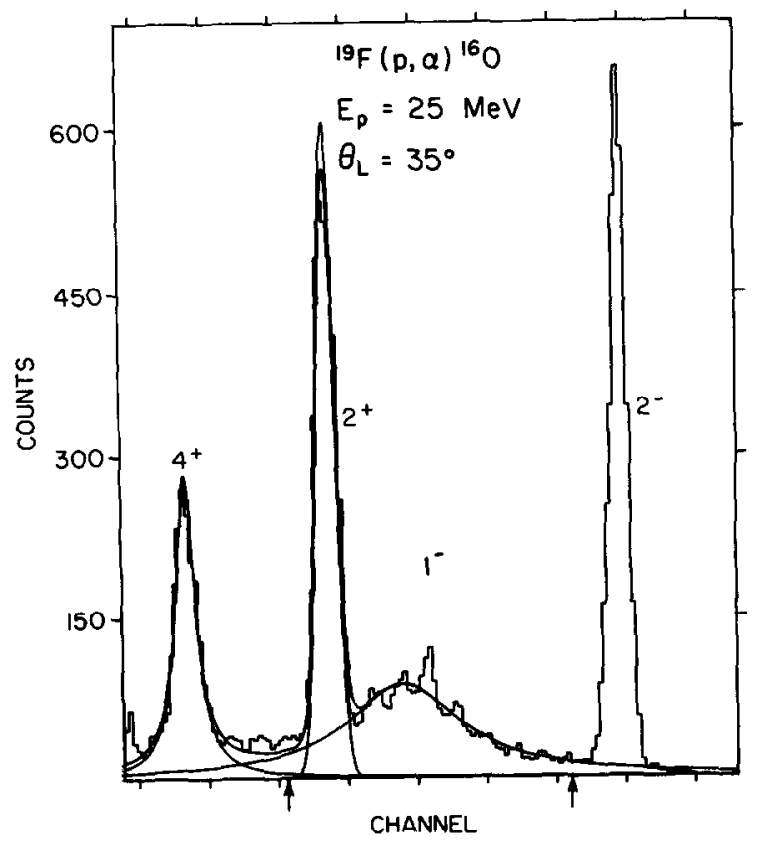

Fig. 7. Same as fig. 2 , for ${ }^{19} \mathrm{~F}(\mathrm{p}, \alpha)^{16} \mathrm{O}$. 
D. Overway et al. / Reaction dependence

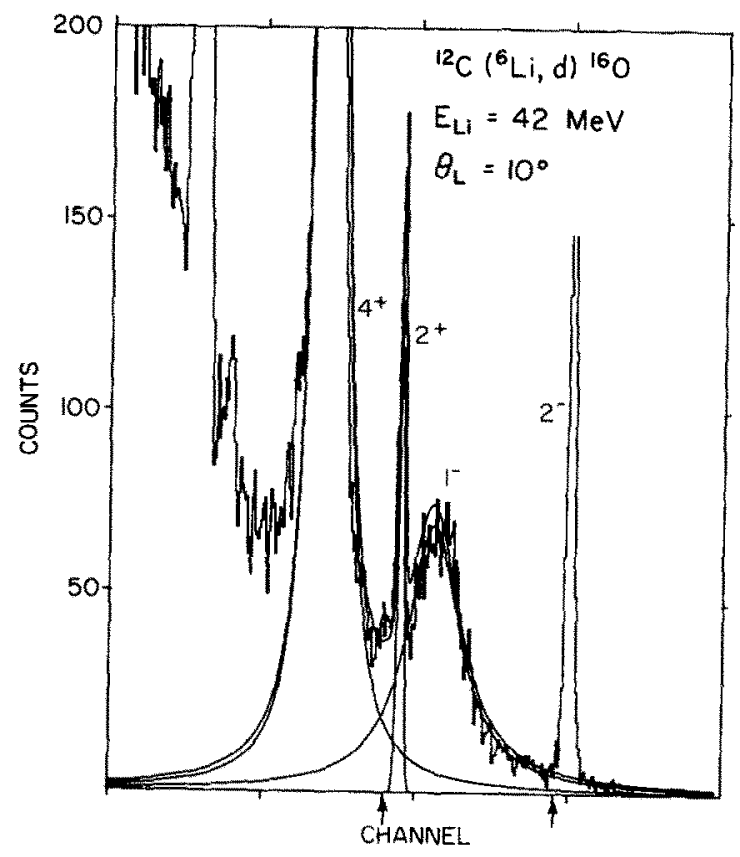

Fig. 8. Same as fig. 2 , for ${ }^{22} \mathrm{C}\left({ }^{6} \mathrm{Li}, \mathrm{d}\right), E(\mathrm{Li})=42 \mathrm{MeV}$.

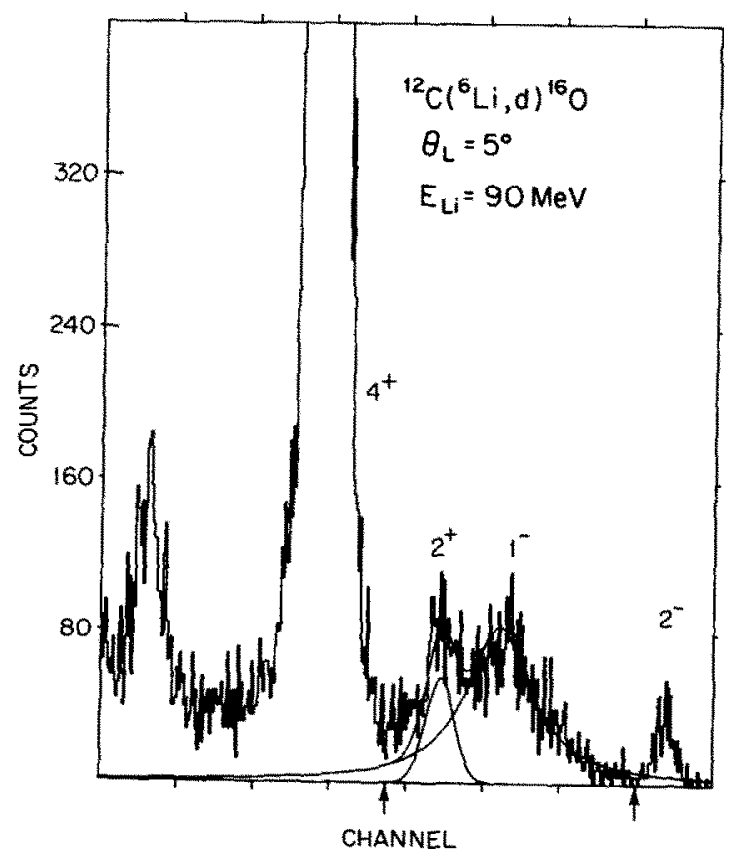

Fig. 9. Same as fig. 2, for ${ }^{12} \mathrm{C}\left({ }^{6} \mathrm{Li}, \mathrm{d}\right), E(\mathrm{Li})=90 \mathrm{MeV}$; data from ref. ${ }^{17}$ ). 


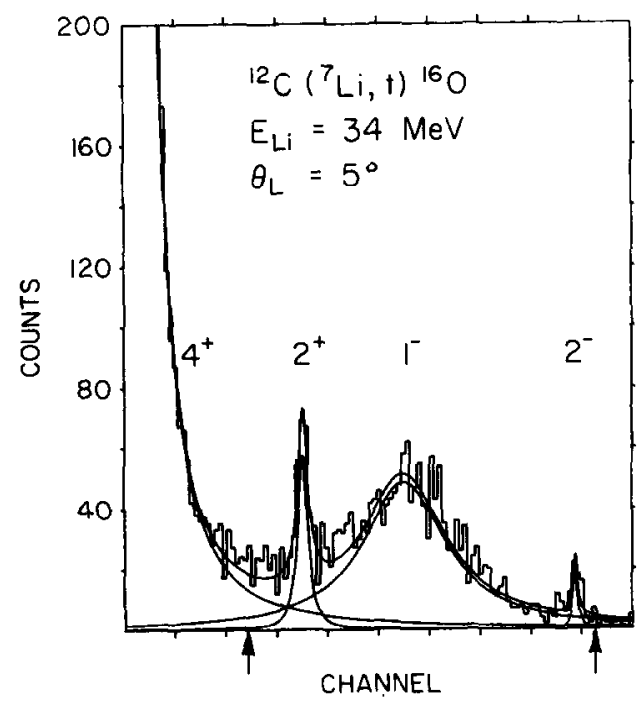

Fig. 10. Same as fig. 2, for ${ }^{12} \mathrm{C}\left({ }^{7} \mathrm{Li}, \mathrm{t}\right)$; data from ref. $\left.{ }^{8}\right)$.

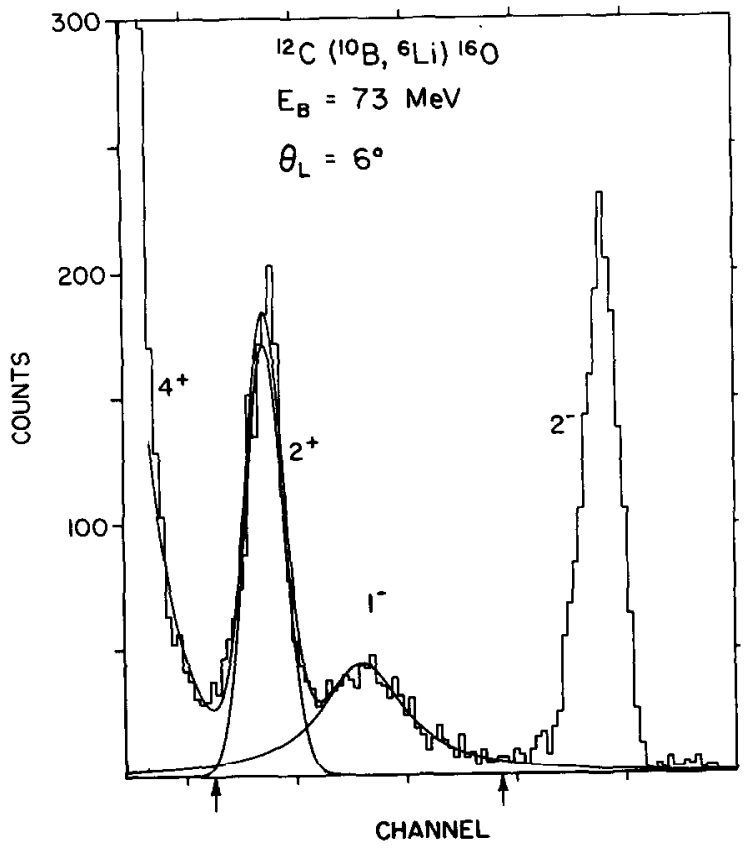

Fig. 11. Same as fig. 2, for ${ }^{12} \mathrm{C}\left({ }^{10} \mathrm{~B},{ }^{6} \mathrm{Li}\right)$. 
from the fitted values of $\Gamma$, if necessary, using the procedures described by Wilkinson [ref. $\left.{ }^{19}\right)$ ]. This was deemed sufficiently accurate for most of the data, considering the uncertainties in the true experimental line shapes which are complicated functions of spectrometer aberrations, energy losses in the target, etc. The data for ${ }^{12} \mathrm{C}^{12} \mathrm{C}$, $\left.{ }^{16} \mathrm{O}\right){ }^{8} \mathrm{Bc}$ required convolution as the experimental resolution was poor (table 1) and it was not possible to extract accurate $\Gamma$-values. It was found that $\Gamma$-values compatible with those obtained from the other data were adequate to fit the data, however.

Typical fits using the form (1) are shown as broken curves in several of the figures. The calculations using fixed, energy-independent widths $(\Gamma)$ are adequate for ${ }^{16} \mathrm{O}^{*}$ $\left(1^{-}, 9.6 \mathrm{MeV}\right)$. Variations in $\Gamma$ for nearly equivalent fits, as determined by the usual chi-squared criterion $\left(\chi^{2} / \mathrm{N}\right)$, can arise from differences in the background shape employed. The latter is attributed to nearby levels in the continuum and phasespace contributions from 3-body processes. This background, relative to the states of intcrest, appears to incrcasc substantially with rcduced bombarding encrgy, i.e. as the reaction becomes less selective and more statistical in nature and the phase space becomes smaller (see sect. 4). This may partly account for the variations observed by Berkowitz ${ }^{4}$ ) although there are more important effects present (subsect. 4.1).

The results obtained for ${ }^{8} \mathrm{Be}^{*}\left(2^{+}, 2.9 \mathrm{MeV}\right)$ are presented in table 2 . Owing to the limited energy range of the spectrometer-detector system, it was necessary to overlap spectra in some instances to obtain a complete excitation-energy spectrum. This introduces some additional uncertainties in the extracted $\Gamma\left(2^{+}, 2.9 \mathrm{MeV}\right)$. In addition the ${ }^{12} \mathrm{C}\left({ }^{12} \mathrm{C},{ }^{16} \mathrm{O}\right){ }^{8} \mathrm{Be}$ reaction has levels excited in the ${ }^{16} \mathrm{O}$ ejectile, which are Doppler-broadened by $\gamma$-decay in flight and contribute substantially to the background continuum (fig. 6).

The simple one-parameter lorentzian line shape $N_{\mathrm{L}}(E)$ does not fit the data and it is necessary to use a more realistic line shape (sect. 4) with an energy-dependent width parameter. Despite this problem, the $\Gamma$-values for ${ }^{8} \mathrm{Be}^{*}$ appear to agree (table 2 ) with the measurements of Nüsslin et al. ${ }^{5}$ ), although different reactions have been employed.

We note the apparent enhancement of counts in the region between the $2.9 \mathrm{MeV}$ level and the ${ }^{8} \mathrm{Be}$ ground state. This will be discussed in subsect. 4.4.

The results for ${ }^{16} \mathrm{O}^{*}\left(1^{-}, 9.6 \mathrm{MeV}\right)$ are given in table 2 . Most $\Gamma$-values for different measurements overlap and indicate no simple reaction dependence.

Additional data as a function of reaction angle were obtained for ${ }^{12} \mathrm{C}\left({ }^{6} \mathrm{Li}, \mathrm{d}\right)$, ${ }^{12} \mathrm{C}\left({ }^{7} \mathrm{Li}, \mathrm{t}\right)$ and ${ }^{19} \mathrm{~F}(\mathrm{p}, \alpha)$. The values of $\Gamma$ for ${ }^{16} \mathrm{O}^{*}\left(1^{-}, 9.6 \mathrm{MeV}\right)$ as a function of $\theta$ are shown in fig. 12. There appears to be perhaps some small ( $\$ 20 \%$ ) correlation between $\Gamma$ and $\theta_{\text {c.m. }}$. particularly for $\left({ }^{7} \mathrm{Li}, \mathrm{t}\right)$ which is relatively free of background at $E_{\mathrm{x}}=9.6 \mathrm{MeV}$ [see ref. $\left.{ }^{8}\right)$ ] compared with other reactions. Perhaps significantly the $\Gamma(9.6 \mathrm{MeV})$ inferred from $(\mathrm{p}, \alpha)$ appears to be somewhat larger than the values obtained from $\left({ }^{6} \mathrm{Li}, \mathrm{d}\right)$ and $\left({ }^{7} \mathrm{Li}, \mathrm{t}\right)$. In addition the analysis of the new data for 
TABLE 2

Summary of decay width analyses

\begin{tabular}{|c|c|c|c|c|c|}
\hline $\begin{array}{l}\text { Nucleus } \\
\left(J^{\pi}, E_{x}\right)\end{array}$ & Reaction & $\begin{array}{c}E_{\text {lab }} \\
(\mathrm{MeV})\end{array}$ & $\begin{array}{c}\left.\Gamma^{\mathrm{a}}\right) \\
(\mathrm{keV})\end{array}$ & $\begin{array}{c}\left.E_{\mathrm{R}}{ }^{\mathrm{b}}\right) \\
(\mathrm{keV})\end{array}$ & $\begin{array}{c}\left.\Gamma_{\mathrm{R}}{ }^{\mathrm{C}}\right) \\
(\mathrm{keV})\end{array}$ \\
\hline${ }^{8} \mathrm{Be}$ & ${ }^{11} \mathrm{~B}(\mathrm{p}, \alpha)$ & 33 & $1300(150)$ & $3260(150)$ & $1640(200)$ \\
\hline \multirow[t]{5}{*}{$\left(2^{+}, 2.9 \mathrm{MeV}\right)$} & ${ }^{9} \mathrm{Be}(\mathrm{p}, \mathrm{d})$ & 33 & $1250(50)$ & $3140(50)$ & $1500(70)$ \\
\hline & ${ }^{9} \mathrm{Be}(\mathrm{d}, \mathrm{t})$ & 26 & $1300(100)$ & $3110(50)$ & $1460(70)$ \\
\hline & ${ }^{12} \mathrm{C}\left(\mathrm{d},{ }^{6} \mathrm{Li}\right)$ & 33 & $1100(100)$ & $3070(150)$ & $1490(150)$ \\
\hline & ${ }^{12} \mathrm{C}\left({ }^{12} \mathrm{C},{ }^{16} \mathrm{O}\right)$ & 80 & $1550(100)$ & $\left.(3150)^{d}\right)$ & $\left.(1550)^{d}\right)$ \\
\hline & Mean & & & $3130(25)$ & $1500(20)$ \\
\hline${ }^{16} \mathrm{O}$ & ${ }^{19} \mathrm{~F}(\mathrm{p}, x)$ & 26 & $450(50)$ & $2408(20)$ & $457(30)$ \\
\hline \multirow[t]{5}{*}{$\left(1^{-}, 9.6 \mathrm{MeV}\right)$} & ${ }^{12} \mathrm{C}\left({ }^{6} \mathrm{Li}, \mathrm{d}\right)$ & 42 & $400(50)$ & $2495(20)$ & $409(25)$ \\
\hline & & 90 & $400(50)$ & $2468(20)$ & $410(25)$ \\
\hline & ${ }^{12} \mathrm{C}\left({ }^{7} \mathrm{Li}, \mathrm{t}\right)$ & 34 & $390(50)$ & $2465(30)$ & $447(40)$ \\
\hline & ${ }^{12} \mathrm{C}\left({ }^{10} \mathrm{~B},{ }^{6} \mathrm{Li}\right)$ & 73 & $350(50)$ & $2447(40)$ & $423(60)$ \\
\hline & Mean & & & $2457(15)$ & $430(10)$ \\
\hline
\end{tabular}

") The quantity $\Gamma$ is the line width defined as the best-fit FWHM utilizing a lorentzian line shape (eq. 1). The latter does not reproduce the ${ }^{8} \mathrm{Be}\left(2^{+}\right)$data (see figs. 2 to 6 ).

b) The resonance energy in the $\alpha+\alpha$ or $\alpha+{ }^{12} \mathrm{C}$ c.m.s. (fig. 1) for the resonance indicated as deduced from the best-fit Breit-Wigner line shape [eq. (6)] utilizing the energy-dependent width $\Gamma(E)$. Does not include a shift function ${ }^{30}$ ).

$\left.{ }^{c}\right)$ The resonance width defined as $\Gamma_{\mathrm{R}}=2 \gamma_{\mathrm{R}}^{2} P_{l}\left(E_{\mathrm{R}}\right)$ inferred from the bestifit Breit-Wigner line shape, eq. (9.6), with $A(E)=0$. The quantities $P_{l}(E)$ are calculated using WKB barrier penetrabilities, $\mathrm{p}_{l}(E)$, obtained with the following nuclear potentials: $\alpha+\alpha\left[\right.$ ref. $\left.\left.{ }^{36}\right)\right]: V=-112 \mathrm{MeV}, R=1.8 \mathrm{fm}, a=0.6 \mathrm{fm} ; \alpha+{ }^{12} \mathrm{C}$ [ref. $\left.\left.{ }^{37}\right)\right]: V=-46 \mathrm{MeV}, R=3.6 \mathrm{fm}, a=0.6 \mathrm{fm}$. The corresponding observed and formal reduced widths and channel radii are $\gamma_{\mathrm{R}}^{2}=580 \pm 50 \mathrm{keV}, \gamma_{\lambda}^{2}=680 \pm 100 \mathrm{keV}(s=4.8 \mathrm{fm})$ and $\gamma_{\mathrm{R}}^{2}=188 \pm 10 \mathrm{keV}, \gamma_{\lambda}^{2}=$ $350 \pm 50 \mathrm{keV}(s=5.4 \mathrm{fm})$, respectively. The formal energies, $E_{\lambda}$, are strongly correlated with $\gamma_{\lambda}^{2}$ and are not well determined (see text). The optimum fits differ slightly from those shown in figs. 2 to 11 , primarily in the analysis of ${ }^{16} \mathrm{O}^{*}\left(4^{+}, 10.3 \mathrm{MeV}\right)$.

d) These values are not well determined, but the data are consistent with other measurements.

${ }^{12} \mathrm{C}\left({ }^{10} \mathrm{~B},{ }^{6} \mathrm{Li}\right)$ confirm the previous measurements and yield a smaller value for $\Gamma\left(1^{-}, 9.6 \mathrm{MeV}\right)$ than does $\left({ }^{6} \mathrm{Li}, \mathrm{d}\right)$.

\subsection{VELOCITY DEPENDENCE}

We have computed the relative velocity of the ejectile ion for the various reactions forming ${ }^{16} \mathrm{O}^{*}\left(1^{-}, 9.6 \mathrm{MeV}\right)$ and ${ }^{8} \mathrm{Be}^{*}\left(2^{+}, 2.9 \mathrm{MeV}\right)$ using the expression

$$
v_{\mathrm{rel}}=\sqrt{2 T_{\mathrm{c} . \mathrm{m}}^{\mathrm{T}} / m_{\mathrm{f}}}
$$

where $T_{c, m}^{\mathrm{f}}$ and $m_{\mathrm{f}}$ are the asymptotic kinetic energy and reduced mass of the ejectile. This expression should be valid for reactions above the Coulomb barrier, such as those reported here, although in principle one should use the local kinetic energy 


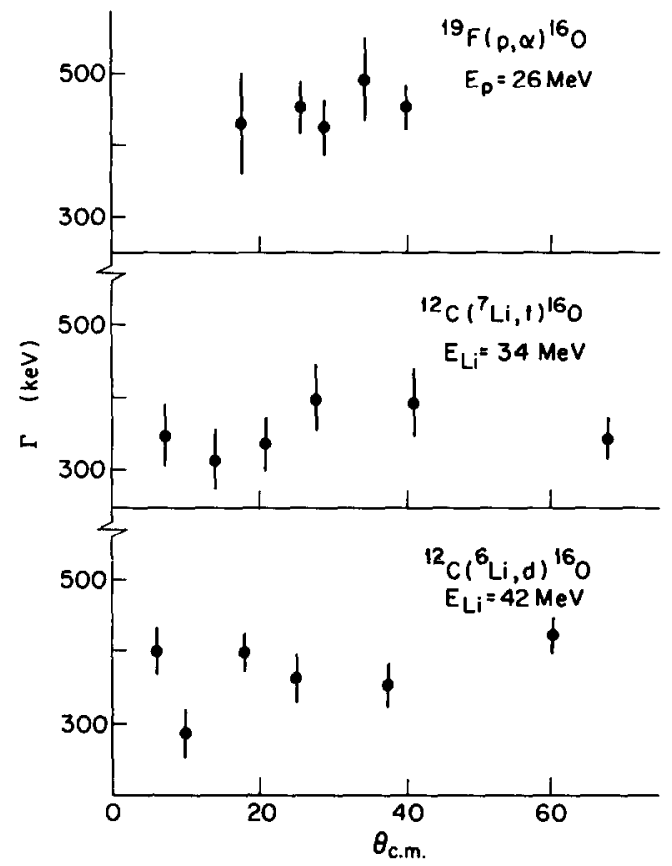

Fig. 12. Variation with scattering angle of the observed width $\Gamma$ for ${ }^{16} \mathrm{O}^{*}\left(1^{-}, 9.6 \mathrm{MeV}\right)$. [Note off-set scales].

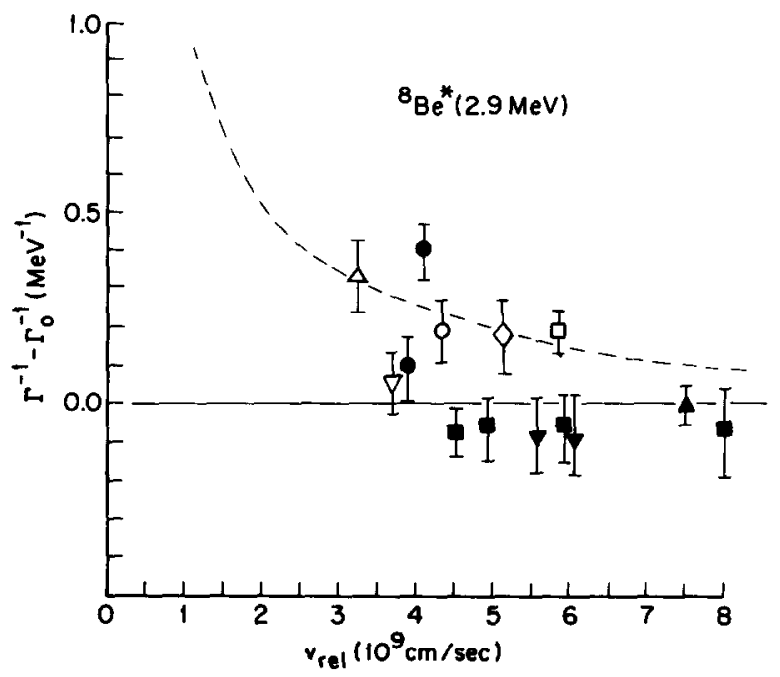

Fig. 13. Variation of $\Gamma^{-1}-\Gamma_{0}^{-1}$ for ${ }^{8} \mathrm{Be}^{*}$ as a function of the asymptotic ejectile c.m. velocity $\left(v_{\text {rel }}\right.$ ). The broken curve represents the behavior $\left(\propto v_{\text {rel }}^{-1}\right)$ given by Berkowitz [ref. $\left.\left.{ }^{4}\right)\right]$. The open symbols represent data from the present experiment: $(\diamond){ }^{11} \mathrm{~B}(\mathrm{p}, \alpha) ;(\Delta){ }^{12} \mathrm{C}\left(\mathrm{d},{ }^{6} \mathrm{Li}\right) ;(\nabla){ }^{12} \mathrm{C}\left({ }^{12} \mathrm{C},{ }^{16} \mathrm{O}\right)$;

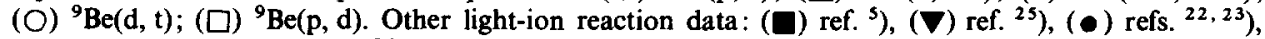
(A) ref. ${ }^{26}$ ). The reference value is taken as $\Gamma_{0}=1.70 \mathrm{MeV}$. 


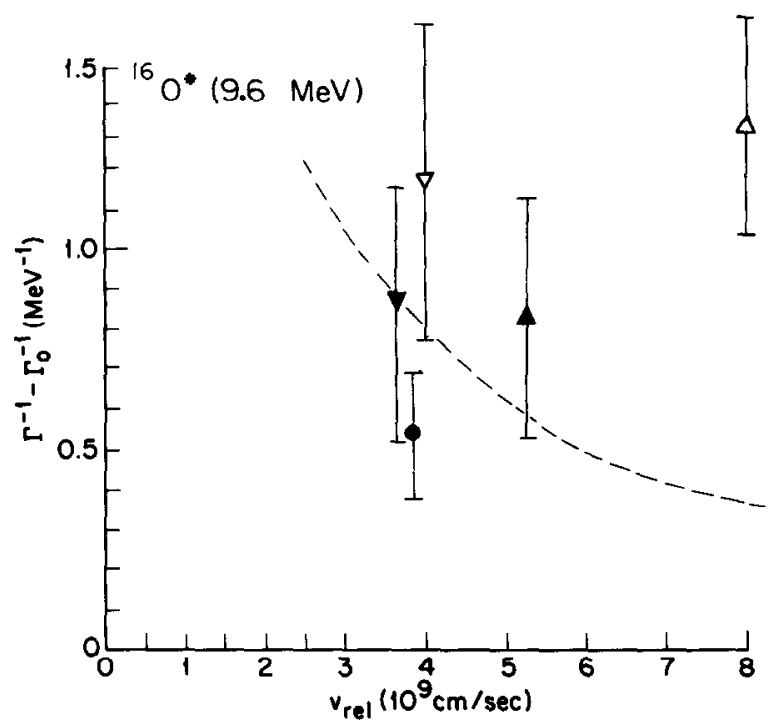

Fig. 14. Same as fig. 13, for ${ }^{16} \mathrm{O}^{*}\left(1^{-}, 9.6 \mathrm{MeV}\right)$; All data are from the present experiment: $(\nabla)\left({ }^{7} \mathrm{Li}, \mathrm{t}\right)$; $(\bullet)^{19} \mathrm{~F}(\mathrm{p}, \alpha) ;(\nabla){ }^{12} \mathrm{C}\left({ }^{10} \mathrm{~B},{ }^{6} \mathrm{Li}\right) ;(\Delta){ }^{12} \mathrm{C}\left({ }^{6} \mathrm{Li}, \mathrm{d}\right), 42 \mathrm{MeV} ;(\triangle){ }^{12} \mathrm{C}\left({ }^{6} \mathrm{Li}, \mathrm{d}\right), 90 \mathrm{MeV}$. The reference value is taken as $\Gamma_{0}=600 \mathrm{keV}$.

$T_{\text {c.m. }}^{\mathrm{f}}(r)=T_{\text {c.m. }}^{\mathrm{f}}(\infty)-V_{\text {Coul }}(r)-V_{\text {nucl }}(r)$. The latter are the Coulomb and nuclear potentials and the radius $r$ corresponds to some appropriate interaction radius, which may depend on $\theta$.

We display in figs. 13 and 14 the quantity $\left(\Gamma^{-1}-\Gamma_{0}^{-1}\right)$ versus $v_{\mathrm{rel}}^{-1}$ for ${ }^{8} \mathrm{Be}^{*}\left(2^{+}\right.$, $2.9 \mathrm{MeV})$ and ${ }^{16} \mathrm{O}^{*}\left(1^{-}, 9.6 \mathrm{MeV}\right)$. The values chosen for $\Gamma_{0}$ are $1700 \mathrm{keV}$ and 600 $\mathrm{keV}$, respectively. Included are data from table 2 as well as some of the previously published line-width data ${ }^{5,20-26}$ ). According to the reaction-time dependence model ${ }^{4}$ ) one would expect a noticeable decrease in $\Gamma^{-1}$ (i.e. increase in $\Gamma$ ) with increasing $v_{\text {rel }}$, as indicated by the broken curve. Instead, the most noticeable effect is a slight difference in $\Gamma$ between some heavy-ion and the light-ion induced reactions. As noted previously the contributions from the continuum are different from these reactions however. Also, the analysis of the ${ }^{14} \mathrm{~N}\left({ }^{3} \mathrm{He}, \mathrm{p}\right)^{16} \mathrm{O}$ data of ref. ${ }^{27}$ ) is complicated by nearby levels and may be more uncertain than the quoted errors would suggest. The results for ${ }^{8} \mathrm{Be}^{*}\left(2^{+}, 2.9 \mathrm{MeV}\right)$ are comparable to those obtained by Nüsslin et al. ${ }^{5}$ ) and Alster et al. ${ }^{21}$ ) and confirm those previously obtained $\left.{ }^{7-9}\right)$ for ${ }^{16} \mathrm{O}^{*}\left(1^{-}, 9.6 \mathrm{MeV}\right)$. Both results indicate no simple dependence, if any, of $\Gamma$ on $v_{\text {rel }}$ although there may be slight differences ( $\$ 20 \%$ ) between $\Gamma$ values inferred with light-ion and heavy-ion reactions.

The anomaly observed for ${ }^{8} \mathrm{Be}^{*}\left(E_{\mathrm{x}} \approx 0.8 \mathrm{MeV}\right.$, figs. 2 to 4$)$ will be discussed in subsect. 4.4. 


\section{Resonance level widths}

\subsection{FACTORS AFFECTING LINE WIDTHS}

The procedure described in subsect. 3.1 neglect several factors which can affect the spectral shape of levels in the continuum. These factors include:

(i) energy dependence of the penetrability of the particle emitted from the decaying state (e.g. ${ }^{8} \mathrm{Be}^{*} \rightarrow \alpha+\alpha$ or ${ }^{16} \mathrm{O}^{*} \rightarrow \alpha+{ }^{12} \mathrm{C}$ );

(ii) phase-space contributions from 3-body, or more complicated events;

(iii) coherent interference between levels of the same spin and parity;

(iv) non-resonant final-state interactions (FSI);

(v) reaction cross-section $Q$-value dependence for the formation of the state.

The penetrability effect (i) is normally included in phase shift analyses of scattering resonances $\left(\alpha+\alpha, \alpha+{ }^{12} \mathrm{C}\right)$ but is often neglected in the extraction of line widths from nuclear reactions. A simple partial-wave resonance line shape can be expressed in terms of the resonant phase shift, $\delta_{l}^{\mathrm{R}}(E)$. A modificd form of the spectral shape has been suggested by Watson ${ }^{13}$ ) which we will denote as $N_{\mathrm{w}}(E)$,

$$
N_{\mathrm{w}}(E) \propto \frac{\sin ^{2} \delta_{l}^{\mathrm{R}}(E)}{k P_{l}(E)} \propto \frac{\sin ^{2} \delta_{l}^{\mathrm{R}}(E)}{k \Gamma(E)},
$$

with

$$
\Gamma(E)=2 \gamma_{\lambda}^{2} P_{l}(E)=2 \gamma_{\lambda}^{2} k s p_{l}(E),
$$

where $\gamma_{\lambda}^{2}$ is the formal reduced decay width (here $\alpha$-decay), $P_{l}(E)$ is the penetrability function, $k$ is the wave number in the decay channel $\left(\alpha+\alpha\right.$ or $\left.\alpha+{ }^{12} \mathrm{C}\right), s$ is the channel radius, and $p_{l}(E)$ is the barrier penetration $\left.{ }^{30}\right)$.

Near a resonance the phase shift is dominated by a term of the form

$$
\delta_{l}^{\mathrm{R}}(E)=\tan ^{-1}\left[\frac{1}{2} \Gamma(E) /\left(E_{\mathrm{R}}^{0}+\Delta(E)-E\right)\right],
$$

and then eq. (3) reduces to a Breit-Wigner expression ${ }^{29,30}$ )

$$
N_{\mathrm{BW}}(E) \propto \frac{\Gamma(E)}{\left(E-E_{\mathrm{R}}\right)^{2}+\left[\frac{1}{2} \Gamma(E)\right]^{2}} \frac{1}{k},
$$

where $E_{\mathbf{R}}=E_{\lambda}+\Delta(E)$ and $\Delta(E)$ is the shift function ${ }^{30}$ ) which depends on $\gamma_{\lambda}^{2}$ and $P_{l}(E)$. It is convenient for practical line-shape analyses to set $\Delta(E)=0$, i.e. exclude the shift function. The corresponding parameters: $E_{\mathrm{R}}, \Gamma_{\mathrm{R}}\left[\equiv \Gamma\left(E_{\mathrm{R}}\right)=2 \gamma_{\mathrm{R}}^{2} P_{l}\left(E_{\mathrm{R}}\right)\right]$ and $\gamma_{R}^{2}$ will be denoted as the observed "resonance" energy, width and reduced width, respectively (see subsect. 4.3). Although the parameter $\Gamma(E)$ is a function of $E$ one notes that $\Gamma \approx \Gamma_{\mathrm{R}}$ for $\Gamma_{\mathrm{R}} / E_{\mathrm{R}} \ll 1$.

Since the barrier penetration is that relevant to the decay channel ( $\alpha$-decay) it is independent of the formation process provided there are no strong final-state interactions. Thus $\Gamma_{\mathrm{R}}, \gamma_{\mathrm{R}}^{2}$ and $\gamma_{\lambda}^{2}$ should be reaction independent in the absence of the latter. 
The phase-space factor (ii) for a reaction $1+2 \rightarrow 3+4 \rightarrow 3+(5+6)$ where 3 is the only observed particle, is given by ${ }^{31,32}$ )

$$
\rho(E) \propto\left[E\left(E_{\max }-E\right)\right]^{\frac{1}{2}},
$$

wherc $E$ is the c.m. cnergy above the threshold for $4 \rightarrow(5+6)$ and $E_{\max }$ is the maximum energy of particle 3 , which depends on the bombarding energy. If particle 3 cannot be distinguished from 5 or 6 , as is the case here for ${ }^{11} \mathrm{~B}(\mathrm{p}, \alpha){ }^{8} \mathrm{Be}$ and ${ }^{19} \mathrm{~F}(\mathrm{p}, \alpha)$ ${ }^{16} \mathrm{O}$ for example, additional terms can arise ${ }^{24,31}$ ) in addition to (7). The phase-space contribution (7) is reaction dependent and can distort line shapes near a decay threshold, as is often the case in elementary particle physics ${ }^{3.31}$ ) and few-nucleon reactions ${ }^{31}$ ).

The line shape given by eq. (6) differs [by various factors of $\Gamma_{\mathrm{R}}(E)$ and $k$ ] from other forms used ${ }^{23,24}$ ) in analyses of decay widths. One notes that in the s-wave limit $P_{l}(E) \propto k(E \gg 0)$ and hence $\Gamma_{\mathrm{R}}(E) \propto k$. Also, one then has $\rho(E) \propto \sqrt{E} \propto k$ since $E_{\max } \gg E$ usually. Thus the product $N_{\mathrm{BW}}(E) \times \rho(E) \rightarrow N_{\mathrm{L}}(E)$, wherc the lattcr is the lorentzian form [eq. (1)] commonly utilized in spectral analyses. Eq. (3) and (6) combined with (7) are generally more valid, however.

The coherence effect (iii) has been discussed by Barker ${ }^{12}$ ) and others. The interference with other levels can narrow or broaden the apparent width of a level depending upon the character of the interference (destructive or constructive) ${ }^{30}$ ). The data for ${ }^{8} \mathrm{Be}^{*}\left(2^{+}, 2.9 \mathrm{MeV}\right)$ and ${ }^{16} \mathrm{O}^{*}\left(1^{-}, 9.6 \mathrm{MeV}\right)$ appear to be reproduced by the simple single-level expression [eq. (6)] and do not exhibit any obvious coherent interference with other levels as observed in some atomic- and nuclear-physics reactions $2,10,11$ ). The only anomaly apparent is the well-known enhancement at $E_{\mathrm{x}} \approx 0.8 \mathrm{McV}$ in ${ }^{8} \mathrm{Bc}^{*}$, which has little cffect on the $\Gamma\left(2^{+}, 2.9 \mathrm{MeV}\right)$ deduced.

The non-resonant background term (iv) represents the decay process $4 \rightarrow(5+6)$ which excludes the resonances described by $\delta_{l}^{\mathrm{R}}(E)$. It may be included as a non-resonant addition to the phase shift $\delta_{l}^{\mathbf{R}}(E)$ of eq. (3) or as a non-coherent background. Final-state interactions may be included if the appropriate scattering phase shifts are known. Alternatively, as is often the case for few-nucleon systems, when only a few partial waves contribute one may deduce $\delta_{1}(E)$ from the spectral shape.

The formation cross-section effect (v) has been estimated previously ${ }^{22,24}$ ) to be on the order of $10 \%$ at moderate bombarding energies $(E \approx 20 \mathrm{MeV})$. This can become more important at low bombarding energies, however, and comparable to (i). It may also introduce an apparent angle-dependent variation in $\Gamma$ (fig. 12) particularly for highly diffractive cross sections, as is often the case for certain direct nuclear reactions. We have estimated this effect using calculations ${ }^{7,8}$ ) which fit the formation cross section angular distributions where available: ${ }^{12} \mathrm{C}\left({ }^{6} \mathrm{Li}, \mathrm{d}\right)$, ${ }^{12} \mathrm{C}\left({ }^{7} \mathrm{Li}, \mathrm{t}\right.$ ), and ${ }^{19} \mathrm{~F}(\mathrm{p}, \alpha)$. The effect on $\Gamma$ (and $E_{\mathrm{R}}$ ), arising from the diffractive structure of $\sigma(E, \theta)$, is $\leqslant 10 \%$ in agreement with refs. $\left.{ }^{23}, 24\right)$. This can account for the slight variations observed in $\Gamma$ as a function of angle.

A review of many of the above features may be found in the articles by Jackson ${ }^{3}$ ) and Slobodrian ${ }^{31}$ ). 


\subsection{ANALYSIS OF RESONANCE LEVELS}

Combining (i), (ii) and (iv) and neglecting (iii) and (v) we adopt the following spectral shape

$$
N(E) \propto \rho(E)\left[\sum_{\mathrm{R}} A N_{\mathrm{BW}}(E)+B\right],
$$

where $A$ is a normalization constant and $B$ represents the sum of non-resonant contributions. The quantity $B$ is assumed to be a constant independent of energy, which is likely valid in a limited region of $N(E)$.

The form of the barrier penetrability (4) is taken as $^{33}$ )

$$
p_{l}(E)=\{1+\exp [f(E)]\}^{-1},
$$

where $f(E)$ is a function of $E$ such that $p_{l}(E) \rightarrow 1(0)$ as $E \rightarrow \infty(0)$ and $p_{l}(E)=0.5$ at $E=E_{\mathrm{B}}$ where $E_{\mathrm{B}}$ is the barrier height. The function $p_{l}(E)$ was initially determined by fitting calculated $\alpha$-penctrabilitics as a function of $E_{\alpha}$. The latter werc obtained from WKB calculations ${ }^{33}$ ) employing realistic (Woods-Saxon) $\alpha$-nucleus potentials similar to those used to fit $\alpha+\alpha$ and $\alpha+{ }^{12} \mathrm{C}$ elastic scattering ${ }^{35,36,37}$ ).

One finds that the $p_{l}(E)$ are not strongly dependent on the choice of channel radius provided the latter is chosen near or inside the classical inner turning point $\left[\right.$ ref. $\left.\left.{ }^{30}\right)\right]$. Also as cxpected nuclear potentials which give similar fits to low energy $\alpha+$ nucleus scattering yield similar barrier penetrabilities. These "realistic" penetrabilities may be simulated by conventional $R$-matrix functions ${ }^{34}$ ) provided one selects a large channel radius for the latter (e.g. $s \approx 6 \mathrm{fm}$ for ${ }^{8} \mathrm{Be}$ ). This procedure corresponds to adjusting the $R$-matrix barrier height (Coulomb potential only) to cqual that of a rcalistic Coulomb plus nuclear potential.

Starting with different forms for $p_{l}(E)$, the width and background parameters,

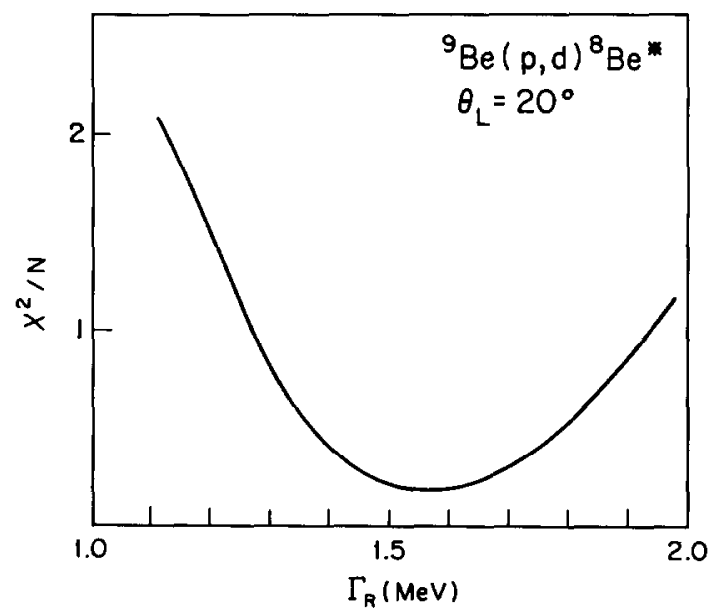

Fig. 15. Typical variation of chi-square versus width parameter for various line-shape fits. 
$\gamma^{2}$ and $B$ respectively, were adjusted to fit the reaction spectra with $p_{l}(E)$ and $s$ kept constant for different reactions populating the same final state. Some of the resulting fits are shown as the solid curves in figs. 2 to 11 . Typical variations in $\Gamma_{\mathrm{R}}$ for ${ }^{8} \mathrm{Be}\left(2^{+}, 2.9 \mathrm{MeV}\right)$ and the corresponding $\chi^{2} / \mathrm{N}$ values are shown in fig. 15 for ${ }^{9} \mathrm{Be}(\mathrm{p}, \mathrm{d})$. The quality of the linc-shape fit is cssentially the same for $1.4 \mathrm{MeV}<\Gamma_{\mathrm{R}}$ $<1.7 \mathrm{MeV}$ depending on the choice of nuclear potentials.

Subsequent calculations employed $p_{l}(E)$ obtained from WKB calculations using a particular set of nuclear potentials ${ }^{36,37}$ ). The resulting width parameters are displayed in table 2. One observes that given a form for $p_{l}(E)$, the resonance parameters $\gamma_{\mathrm{R}}^{2}, \Gamma_{\mathrm{R}}$ and $E_{\mathrm{R}}$ exhibit little variation between different reactions $(\leqslant 10 \%)$ leading to the same final state. Typically (table 2) one finds $\Gamma<\Gamma_{\mathrm{R}}$ with differences on the order of $20 \%$ for ${ }^{8} \mathrm{Be}^{*}\left(2^{+}, 2.9 \mathrm{MeV}\right)$.

After analysis without a shift function, $\Delta(E)$, the latter was included in the fitting procedure and the formal parameters $\left.{ }^{30}\right) E_{\lambda}$ and $\gamma_{\lambda}^{2}$ were determined. These were found to be strongly correlated and $E_{\lambda}$, unlike $E_{\mathrm{R}}$, is not well determined.

\subsection{COMPARISON WITH $R$-MATRIX ANALYSES}

The resonance parameters $E_{\mathrm{R}}, \Gamma_{\mathrm{R}}$ and $\gamma_{\mathrm{R}}^{2}$ [defined for $\Delta(E)=0$ ] are usually well determined by the observable centroid energies and line widths $\left(E_{\mathrm{c}}\right.$ and $\left.\Gamma\right)$ whereas the quantities $E_{\lambda}, \Gamma_{\lambda}$ and $\gamma_{\lambda}^{2}$ of formal $R$-matrix theory ${ }^{30}$ ) often are not. The formal energies and widths are very model dependent and depend on the choice of channel radius and nuclear potential i.e. penetrability. Thus caution must be exercised when comparing formal widths even for the same choice of channel radii.

The distinction between formal and observed widths is crucial for states near threshold since then $\Gamma<\Gamma_{\mathrm{R}} \Gamma_{\lambda}$. Unfortunately this distinction is not always made, particularly when comparing widths obtained from nuclear reactions $\left(\Gamma\right.$ or $\left.\Gamma_{\mathrm{R}}\right)$ with those from scattering resonances $\left(\Gamma_{\lambda}\right)$.

A comparison of our formal reduced widths (table 2) with those obtained from analyses of resonance scattering $\left.{ }^{6}\right)$ for ${ }^{8} \mathrm{Be}(\alpha+\alpha)$ indicates satisfactory agreement, i.e. within $\pm 20 \%$ for the same choice of channel radius. Our $\gamma_{\lambda}^{2}$ values for ${ }^{16} \mathrm{O}^{*}$ $\left(9.6 \mathrm{MeV}, 1^{-}\right)$still appear to be less $($by $\approx 100 \mathrm{keV})$ than values obtained ${ }^{6,20}$ ) from $\alpha+{ }^{12} \mathrm{C}$ scattering although the present values (table 2 ) are reasonably self-consistent (see subsect. 4.5).

\subsection{GHOST STATES}

Enhancements in the continuum just above the ${ }^{8} \mathrm{Be}$ (g.s.) were observed for ${ }^{9} \mathrm{Be}(\mathrm{d}, \mathrm{t}),{ }^{9} \mathrm{Be}(\mathrm{p}, \mathrm{d})$ and to a lesser degree in ${ }^{11} \mathrm{~B}(\mathrm{p}, \alpha)$ [figs. 2 to 4$]$. The model proposed ${ }^{12}$ ) for this phenomenon predicts that the intensity of the "ghost" (as percent of g.s. per $\mathrm{MeV}$ excitation energy) should be independent of bombarding energy and angle, as well as reaction provided the effects (ii) and (v) are small. Barker's 
analyses ${ }^{12}$ ) give a ghost intensity of about $10 \%$ per $\mathrm{MeV}$ at $E_{\mathrm{x}} \approx 0.8 \mathrm{MeV}$. In contrast, three-body threshold effects which have been proposed as an alternate explanation ${ }^{15,16}$ ) would change substantially with bombarding energy, angle, and reaction.

We display in figs. 2 and 3 the regions of interest for ${ }^{8} \mathrm{Bc}^{*}$. The intensity of the ghost state has been extracted from our ${ }^{9} \mathrm{Be}(\mathrm{p}, \mathrm{d})$ and ${ }^{9} \mathrm{Be}(\mathrm{d}, \mathrm{t})$ data by subtracting out the contribution from ${ }^{8} \mathrm{Be}^{*}\left(2^{+}, 2.9 \mathrm{MeV}\right)$ as determined from the line shape analysis: The results are shown in fig. 16 together with predictions by Barker et al. ${ }^{12}$ )

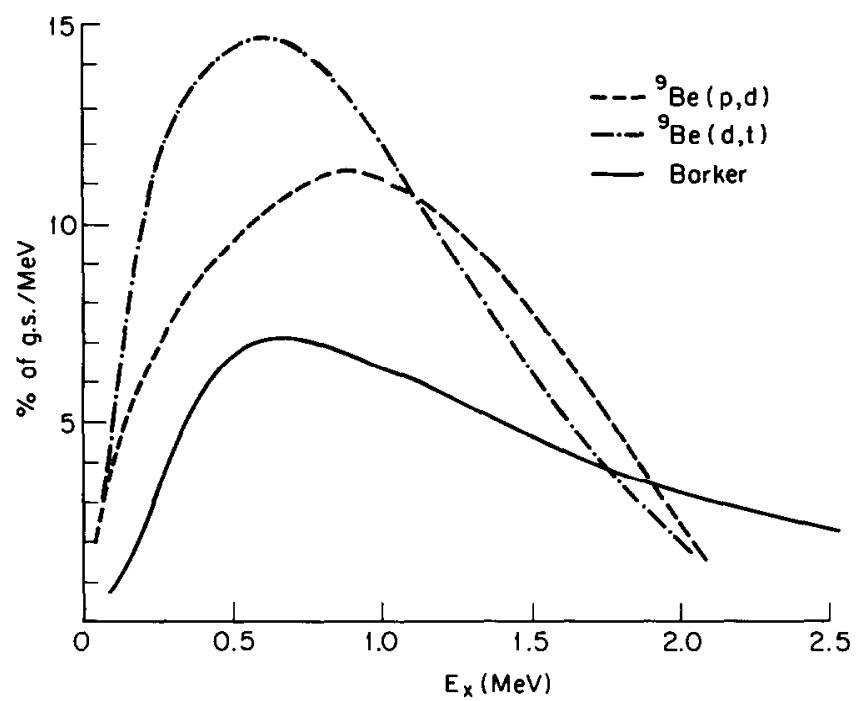

Fig. 16. The shape of the anomaly in ${ }^{8} \mathrm{Be}^{*}$ near $E_{\mathrm{x}} \approx 0.8 \mathrm{MeV}$ compared with the calculation of Barker et al. ${ }^{12}$ ).

based on analysis of other data at lower bombarding energies. The latter are in qualitative agreement with the shape and magnitude of the present data. The $(p, d)$ and $(d, t)$ data exhibit differences in the excitation energy of the maxima of the ghost state $(0.6 \mathrm{MeV}$ versus $0.8 \mathrm{MeV})$ as well as slight differences in magnitude.

Analysis of the data for ${ }^{12} \mathrm{C}\left(\mathrm{d},{ }^{6} \mathrm{Li}\right),{ }^{11} \mathrm{~B}(\mathrm{p}, \alpha)$ and ${ }^{12} \mathrm{C}\left({ }^{12} \mathrm{C},{ }^{16} \mathrm{O}\right)$ are consistent with an upper limit of $15 \%$ of g.s. per $\mathrm{MeV}$ for the ghost state intensity in ${ }^{8} \mathrm{Be}^{*}$ at $E_{\mathrm{x}} \approx 0.8$ $\mathrm{MeV}$. The analysis of this effect is complicated by the presence of background in the ghost region. This background arises from the non-resonant phase-space continuum (ii) and (iv) and contributions from other resonant states ${ }^{20,38}$ ) at $E_{\mathrm{x}}>2.9$ $\mathrm{MeV}$ (see fig. 1).

We have fit the anomaly in ${ }^{8} \mathrm{Be}$ using a one-level resonance for the ${ }^{8} \mathrm{Be}$ (g.s.). This, together with a small phase space background is adequate to reproduce the observed data (figs. 2 to 6 ). The data at $E_{\mathrm{x}} \approx 0.8 \mathrm{MeV}$ cannot be reproduced with simple phase-space alone ${ }^{15,16}$ ) and supports Barker's interpretation of this feature. 
Recent measurements of ${ }^{9} \mathrm{Be}(\mathrm{p}, \mathrm{d})$ at $E_{\mathrm{p}}=26 \mathrm{MeV}$ also appear to confirm this ${ }^{39}$ ). The data for ${ }^{16} \mathrm{O}^{*}$ do not indicate any obvious anomalies (figs. 7 to 11 ). The analysis is limited by the presence of trace amounts of ${ }^{13} \mathrm{C}$ in the targets, however (table 1).

Near particle decay thresholds one might expect to observe featurcs similar to that seen for ${ }^{8} \mathrm{Be}^{*}$. These will exhibit characteristics of true nuclear states including spin, parity, etc. ${ }^{39}$ ). One may need to consider such threshold effects when interpreting broad structures observed in the continuum, e.g. in heavy-ion reactions.

\subsection{REDUCED ALPHA WIDTHS}

The reduced $\alpha$-widths of ${ }^{8} \mathrm{Be}\left(0^{+}\right.$, g.s. $),{ }^{8} \mathrm{Be}^{*}\left(2^{+}, 2.9 \mathrm{MeV}\right)$ and ${ }^{16} \mathrm{O}^{*}\left(1^{-}, 9.6 \mathrm{MeV}\right)$ are important in nuclear astrophysics as they influence the ${ }^{8} \mathrm{Be} \rightarrow{ }^{12} \mathrm{C}$ and ${ }^{12} \mathrm{C} \rightarrow{ }^{16} \mathrm{O}$ $\alpha$-capture rates during stellar helium burning.

An analysis of ${ }^{8} \mathrm{Be}$ (g.s.) and the ghost anomaly will appear elsewhere. The reduced $\alpha$-widths determined for ${ }^{8} \mathrm{Be}^{*}\left(2^{+}, 2.9 \mathrm{MeV}\right)$ from $\Gamma_{\mathrm{R}}$ are $\gamma_{\mathrm{R}}^{2}=580 \pm 50 \mathrm{keV}$ and $\gamma_{\lambda}^{2}=680 \pm 100 \mathrm{keV}(s=4.8 \mathrm{fm}$ ) for our adopted $\alpha$-nucleus potential (table 2 ). This corresponds to $\theta_{\lambda}^{2}=\gamma_{\lambda}^{2} / \gamma_{\mathrm{W}}^{2}=0.50 \pm 0.07$ where $\gamma_{\mathrm{W}}^{2}$ is the Wigner limit ${ }^{34}$ ).

The reduced $\alpha$-widths for ${ }^{16} \mathrm{O}^{*}\left(1^{-}, 9.6 \mathrm{MeV}\right)$ deduced from our $\Gamma_{\mathrm{R}}$ values (table 2) are $\gamma_{R}^{2}=188 \pm 10 \mathrm{keV}$ and $\gamma_{\lambda}^{2}=350 \pm 50 \mathrm{keV}(s=5.4 \mathrm{fm})$. This corresponds to $\theta_{\lambda}^{2}=0.49 \pm 0.07$ which is smaller than the value determined from a recent analysis ${ }^{37}$ ) of $\alpha+{ }^{12} \mathrm{C}$ scattering $\left(\theta_{\lambda}^{2}=0.94\right)$.

The authors thank F. C. Barker, Y. Iwasaki, H. Lipperheide, K. Fuchs, H. Homeyer and F. Slobodrian for suggestions and comments. One of us (F.B.) thanks the staff of the Hahn-Meitner Institute for their hospitality while on a research leave there.

\section{References}

1) R. G. Breene, Jr., The shift and shape of spectral lines (Pergamon, NY, 1961)

2) U. Fano, Phys. Rev. 124 (1961) 1866

3) J. D. Jackson, Nuovo Cim. 34 (1964) 1644

4) E. H. Berkowitz, Nucl. Phys. 60 (1964) 555

5) F. Nüsslin, J. Zimmerer, K. W. Maris and H. Werner, Nucl. Phys. A123 (1969) 300

6) F. Ajzenberg-Selove, Nucl. Phys. A281 (1977) 1 ; A320 (1979) 1

7) F. D. Becchetti, J. Jänecke and C. E. Thorn, Nucl. Phys. A305 (1978) 313

8) F. D. Becchetti, E. R. Flynn, D. L. Hanson and J. W. Sunier Nucl. Phys. A305 (1978) 293

9) F. D. Becchetti et al., in Clustering aspects of nuclear structure and nuclear reactions, ed. W. van Oers et al., (AIP, New York, 1978) p. 752

10) H. Fuchs et al., Nucl. Phys. A196 (1972) 296; J. of Phys. G5 (1979) 797; J. Bommer, et al., Phys. Rev. C12 (1975) 1069

11) G. Baur, F. Roesl and D. Trautmann, J. of Phys. G4 (1978) 275

12) F. C. Barker and P. B. Treacy, Nucl. Phys. 38 (1962) 33:

H. J. Hay et al., Austral I Phys. 20 (1967) 59:

F. C. Barker el al., Austral. J Phys. 21 (1968) $239: 22$ (1969) 293

13) K. M. Watson, Phys. Rev. 88 (1952) 1163

14) F. C. Barker, G. M. Crawley, P. S. Miller and W. F. Steele, Austral. J. Phys. 29 (1976) 245 
15) E. H. Berkowitz, G. L. Marolt, A. A. Rollefson and C. P. Browne, Phys. Rev. C4 (1971) 1564

16) Th. Lorenz, Naturf. 21a (1966) 1196

17) F. D. Becchetti, D. Overway, J. Jänecke and W. W. Jacobs, Nucl. Phys. A344 (1980) 336

18) M. Anderson, program IGPLOT (University if Michigan, 1978) unpublished

19) D. H. Wilkinson, Nucl. Inst. 95 (1971) 259

20) F. Ajzenberg-Selove and T. Lauritzen Nucl. Phys. A227 (1974) 1

21) J. Alster and W. G. Weitkamp, Nucl. Phys. Laboratory Annual Report (University of Washington, 1966) unpublished, p. 32

22) R. Roy, J. Birchall and R. J. Slobodrian, Phys. Lett. 34B (1971) 491

23) R. Roy et al., Nucl. Phys. A245 (1975) 87

24) K. Fukunaga et al., J. Phys. Soc. Japan 42 (1977) 725

25) C. H. Johnson and C. C. Trail, Phys. Rev. 133 (1964) B1183

26) S. Kato et al., Annual Report (INS, Tokyo, 1969) unpublished, p. 13

27) C. P. Browne and I. Michael, Phys. Rev. 134 (1964) B133

28) G. C. Phillips, Ta. S. Griffy and L. C. Biedenharn, Nucl. Phys. 21 (1966) 327

29) E. P. Wigner and L. Eisenbud, Phys. Rev. 72 (1947) 29

30) A. M. Lane and R. G. Thomas, Rev. Mod. Phys. 30 (1958) 257

31) R. J. Slobodrian, Rep. Prog. Phys. 34 (1971) 175

32) G. G. Ohlsen, Nucl. Instr. 37 (1965) 240;

G. G. Ohlsen and P. G. Young, Phys. Rev. 136 (1964) B1632

33) N. Fröman and P. Fröman, JWK B approximation (North-Holland, Amsterdam, 1965)

34) J. B. Marion and F. C. Young, Nuclear reaction analysis (North-Holland, Amsterdam, 1968) ch. IV

35) C. M. Perey and F. G. Perey, Atomic and Nuclear Data Tables 17 (1976) 80

36) G. Igo, Phys. Rev. 117 (1960) 1079

37) S. E. Koonin, T. A. Tombrello and G. Fox, Nucl. Phys. A220 (1974) 221

38) L. A. Kull, Phys. Rev. 163 (1967) 1066

39) F. D. Becchettı et al., submitted for publication 